\title{
Cellular Automata for Electrochemistry. Implementation of Uniform Flows \\ Shigeo HAYASHI*
}

\author{
Department of Engineering Science, University of Electro-Communications, \\ 1-5-1 Chofugaoka, Chofu, Tokyo 182-8585, Japan
}

*Corresponding author: hays@pc.uec.ac.jp

\section{ABSTRACT}

One-dimensional electrochemical cellular automaton (1D-ECA) has been extended so that effects of axial uniform flows toward the electrode can be simulated. The model is characterized by a sink connected to the terminating cell and a pair of asymmetric propagation probabilities that enables a drift with a constant velocity toward the electrode. The plots of the reciprocal stationary-state current and the reciprocal flow velocity yield a line, whose intercept corresponds to the current in the limit of fast mass transport.

(c) The Electrochemical Society of Japan, All rights reserved.

Keywords : Cellular Automata, Computer Simulation, Fluid Flow, Stationary-state Current

\section{Introduction}

A common, practicable method for characterizing the catalytic activity of electrode ${ }^{1}$ is to measure the electrical current at the electrode potential that is adequate for the reaction in question, i.e., to apply chronoamperometry to the system. In general the larger the current or its duration, the better the electrode is.

This may seem a matter of course, but some students of electrochemistry might ask why the current does not decay to zero in proportion to the reverse square root of time. This current, often called the Cottrel current, ${ }^{2}$ is observed, no matter how active the electrode may be. The decay is accounted for by diffusion, which tends to cease supplying reactants to the electrode.

An appropriate answer is that we should take into account masstransport mechanisms that supersede diffusion such as fluid flow. But the students might further ask what difference that kind of mass transport makes from diffusion, which is also a kind of mass transport.

Thus, whether or not the stationary-state current, i.e., the current after a sufficiently long period of time, is finite should depend on the nature of mass transport. Furthermore, this current, hereafter denoted by $I_{\mathrm{S}}$, is not necessarily a good indicator of catalytic activity, since $I_{\mathrm{S}}$ results from an interplay between the mass transport and the reaction rate on the electrode; there should be no clear transition between finite and zero $I_{\mathrm{S}}$ 's.

One-dimensional electrochemical cellular automaton (1D$\mathrm{ECA})^{3-7}$ is an algorithm devised originally for diffusion ${ }^{8}$ and extended to electron transfer. A feature of diffusion is that mass supply tends to vanish together with the concentration gradient, and therefore in order for $I_{\mathrm{S}}$ to be finite some kind of mass transport ought to be incorporated. Examples are fluid flows and finite diffusion lengths. The latter is a straightforward extension of 1DECA, but the former is a challenging problem.

It is thus a primary purpose of the paper to extend 1D-ECA so that fluid flow can be treated. In general fluid flow is a threedimensional phenomenon and is therefore beyond the framework of one-dimensional models. However, it should be possible to deal with a uniform flow, one of the simplest flows, in which streamlines are distributed homogeneously in space. The uniform flow is blocked by an electrode of finite dimensions, and it is assumed that the disturbance on the electrode surface is minimal; in the language of 1D-ECA only a terminating cell that contacts with the electrode is affected, while the remaining cells follow the same algorithm.

If electrochemistry is not involved, this type of mass transport can be dealt with in terms of scalar 1D-ECA, and commercial spreadsheet programs can be utilized for small-scale simulations. This is a unique feature of 1D-ECA as compared with the conventional computer simulation. ${ }^{9,10}$ Since at least two chemical species participate in electrochemistry, 1D-ECA must be in the vector form. It will turn out that incorporating the above-mentioned hydrodynamical consideration into 1D-ECA necessitates only a minor modification in the algorithm, and numerical calculations are expected to yield finite $I_{\mathrm{S}}$ 's.

\section{One-dimensional Electrochemical Cellular Automaton}

\subsection{Basic algorithm}

The methodology has been described elsewhere, ${ }^{3-7}$ and therefore a brief summary is presented here except for new features. We consider the following quasireversible reaction ${ }^{2}$

$$
\mathrm{Ox}+\mathrm{e}^{-} \rightleftharpoons \mathrm{Rd} \text {. }
$$

1D-ECA accounts separately for two processes, mass transport and electron transfer. Mass transport is explained in terms of the vector $\boldsymbol{P}_{i}^{k}$, composed of the concentrations of the two species at time $t=k \delta \mathrm{t}$ and position $x=i \delta x$,

$$
\boldsymbol{P}_{i}^{k}=\left(\begin{array}{c}
P(\mathrm{Ox}) \\
P(\mathrm{Rd})
\end{array}\right)
$$

where $P(\mathrm{Ox})$ and $P(\mathrm{Rd})$ are the concentrations of oxidized $(\mathrm{Ox})$ and reduced $(\mathrm{Rd})$ forms, respectively. The vector satisfies the recursive relation $^{3,8}$

$$
\boldsymbol{P}_{i}^{k+1}=q_{i-1} \boldsymbol{P}_{i-1}^{k}+p_{i+1} \boldsymbol{P}_{i+1}^{k}, \quad\left(1 \leq i \leq N_{x} ; 0 \leq k \leq N_{t}\right)
$$

where the propagation probabilities $p_{i}$ and $q_{i}$, satisfying

$$
p_{i}+q_{i}=1
$$

are allowed to depend on $i$, but will be more restricted in the subsequent sections. In fact the previous treatments used $p_{0}=q_{0}=$ $1 / 2$ for all values of $i$.

Electron transfer, on the other hand, is governed by 


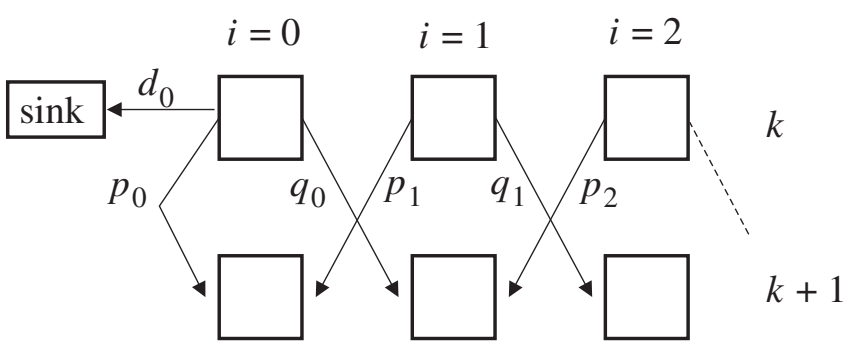

Figure 1. Evolution of cellular automaton that is incorporated with a sink for mass transport.

$$
\boldsymbol{P}_{0}^{k+1}=p_{0} \boldsymbol{A} \boldsymbol{P}_{0}^{k}+p_{1} \boldsymbol{P}_{1}^{k}-d_{0} \boldsymbol{P}_{0}^{k}
$$

where $\boldsymbol{A}$ is a $2 \times 2$ transition matrix since there are two components within the solution. The components of $\boldsymbol{A}$-matrix are transition probabilities $r_{\mathrm{f}}$ and $r_{\mathrm{b}}$,

$$
\boldsymbol{A}=\left(\begin{array}{cc}
1-r_{\mathrm{f}} & r_{\mathrm{b}} \\
r_{\mathrm{f}} & 1-r_{\mathrm{b}}
\end{array}\right) \text {. }
$$

The transition probabilities obey the following expressions,

$$
\begin{aligned}
& r_{\mathrm{f}}(\varepsilon)=\frac{v K_{R O}}{1+K_{R O}} \frac{1}{1+\exp \left(n \varepsilon / V_{T}\right)} \\
& r_{\mathrm{b}}(\varepsilon)=\frac{v}{1+K_{R O}} \frac{1}{1+\exp \left(-n \varepsilon / V_{T}\right)},
\end{aligned}
$$

where $v$ is an enhancement factor, which should not be confused with the number of electrons transferred $n$ or the sweep rate $v$, $K_{R O}$ an asymmetry parameter between reduction and oxidation rates. Both of $n$ and $K_{R O}$ are kept at unity throughout the paper. $V_{T}$, defined by (Boltzmann constant) $\times$ (temperature) $/$ (elementary charge), takes the value $25 \mathrm{mV}$ at the room temperature, ${ }^{3} \varepsilon$ the overpotential defined by

$$
\varepsilon=E-E_{\mathrm{eq}},
$$

$E_{\text {eq }}$ being the equilibrium potential at which no electrical current flows for standard concentrations of $\mathrm{Ox}$ and $\mathrm{Rd}$.

The parameter $d_{0}$, which is introduced for the first time in the present paper, specifies the rate of transport of Ox and Rd to the sink connected to the cell at $i=0$, and the most appropriate value of $d_{0}$ is sought in the next section. The algorithm of time evolution is illustrated in Fig. 1.
In the present paper, a number of calculations will be made while the value of $v$ is varied. The larger the value of $v$, the larger the value of the standard rate constant $k_{\mathrm{s}},{ }^{5}$ but it is difficult to specify how the two are related to each other. As a rule of thumb, $v=1$ means fast electron transfer and $v=0.01$ slow electron transfer. In the case of fuel cells, for example, the electrode with $v=1$ should deserve to be called a good catalytic electrode but the electrode with $v=0.01$ is a poor one.

Finally, the electrical current is proportional to the rate of net electron transfer to and from the electrode, and the unscaled value is given by (see Eq. (8) of the preceding paper $^{3}$ )

$$
I^{k}=n p_{0}\left[r_{\mathrm{f}}(\varepsilon) P_{0}^{k}(\mathrm{Ox})-r_{\mathrm{b}}(\varepsilon) P_{0}^{k}(\mathrm{Rd})\right],
$$

which is adopted throughout this paper, but it can be transformed to the observed current density $J_{\text {obs }}^{k}$ by

$$
J_{\mathrm{obs}}^{k}=a F\left(\frac{\delta x}{\delta t}\right) I^{k},
$$

where $F$ is the Faraday constant and $a$ an appropriate numerical factor that depends on the units of concentration and the current density. If the concentration is measured in units of $\mathrm{mol} / \mathrm{L}$ and the current density in units of $\mathrm{A} / \mathrm{cm}^{2}$, then $a=0.1$, provided that the remaining parameters are expressed in SI units.

\subsection{Implementation of a uniform flow}

A common flow in electrochemistry is the one caused by the rotating disk electrode (RDE), which revolves at an angular velocity of $\omega$, inducing a flow toward the electrode. The flow is complicated since the flow velocity varies with the position. ${ }^{11}$ Our aim is to mimic the flow within a one-dimensional model, and as a first step to this goal the real flow is simplified as shown in Fig. 2(a), where a uniform flow of a constant velocity $V$ is blocked by a disk electrode. There still exists a radial flow near the surface, causing a density distribution along the streamlines over the surface, and therefore let us further simplify the model as shown in Fig. 2(b), where the uniform flow is "cut" on the surface. The terminating streamlines cannot occur in the real flow, but can be realized by introducing a sink for mass transport at the end of streamlines. The onedimensional flow can be treated by the 1D-ECA of Fig. 2(c), where a sink is attached to the cell at $i=0$ on the electrode. The cell at $i=N_{x}$ plays a role of the source, maintaining indefinitely the initial concentration but is of little importance if it is located far away from the electrode and if the number of iterations is relatively small; the (a)

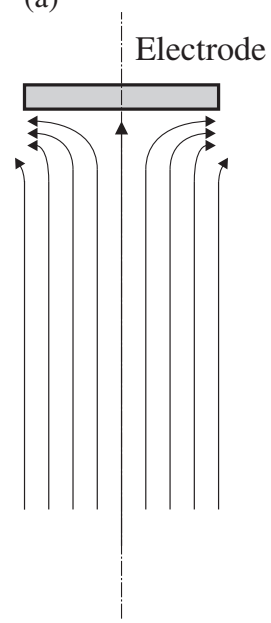

(b)

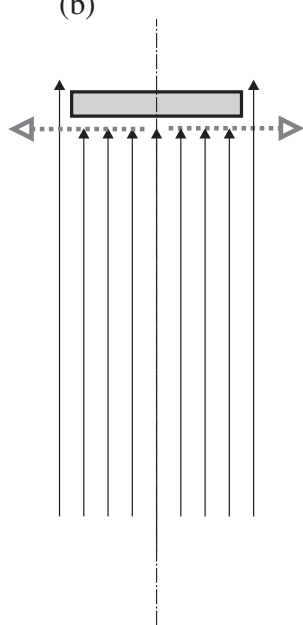

(c)

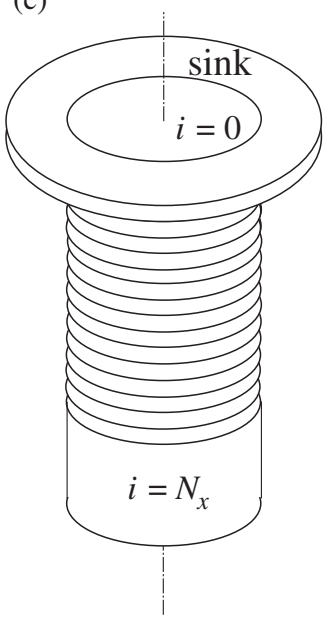

Figure 2. Fluid flow originating from a uniform flow. (a) Schematic representation of simplified 3-D flow. (b) Reduction to 1-D flow, with hollow arrows representing a sink that enables a stationary distribution of chemical species along the flow. (c) 1D-ECA with a sink for mass transport. 
source located close to the electrode may provide a finite flux of reactants, as will be shown in Fig. 11 below.

In order to construct an algorithm for the 1D-ECA of Fig. 2(c), we first note that the sum of the concentrations $X$ defined by

$$
X_{i}^{k}=P_{i}^{k}(\mathrm{Ox})+P_{i}^{k}(\mathrm{Rd})
$$

is independent of the electron-transfer probabilities for any value of $i$, and thus the quantity $X_{i}^{k}$ describes only the transport behavior. In fact, Eqs. (5) and (6) give a scalar equation for the boundary condition

$$
X_{0}^{k+1}=p_{0} X_{0}^{k}+p_{1} X_{1}^{k}-d_{0} X_{0}^{k}
$$

and Eq. (3) leads to a set of recursive scalar equations

$$
X_{i}^{k+1}=q_{i-1} X_{i-1}^{k}+p_{i+1} X_{i+1}^{k} . \quad\left(1 \leq i \leq N_{x} ; 0 \leq k \leq N_{t}\right)
$$

Next, the constant flow velocity is shown to dictate the values of $p_{i}$ and $q_{i}$. For this purpose, let us assume that the spatial and temporal step sizes, $\delta x$ and $\delta t$, are so small that differences can be approximated by differentials. Then we are led to the following onedimensional convective diffusion equation

$$
\frac{\partial X}{\partial t}=D \frac{\partial^{2} X}{\partial x^{2}}-\frac{\partial(V x)}{\partial x},
$$

where $X \equiv X(x, t)$ is an extension of $X_{i}^{k}$, which has been defined only at discreet points, to continuous space, $D$ is the diffusion coefficient defined by

$$
D=\frac{(\delta x)^{2}}{2 \delta t}
$$

and $V$ has the meaning of the drifting velocity or the flow velocity given by

$$
V=(p-q) \frac{\delta x}{\delta t}
$$

In the present paper, the flow is assumed to have a constant velocity irrespective of the position along the axis, meaning that $(p-q)$ has the same value everywhere. The propagation probabilities therefore can be set to the following forms in terms of the asymmetric propagation parameter $g$,

$$
\begin{aligned}
p_{i} & =\frac{1}{2}(1+g), \\
q_{i} & =\frac{1}{2}(1-g),
\end{aligned}
$$

for $i \geq 0$. The fluid velocity is thus proportional to $g$,

$$
V=g \frac{\delta x}{\delta t}
$$

It remains to determine the value of the sink parameter $d_{0}$, which should be related to $g$ in some way. This task can be carried out from numerical tests such that the behavior at the electrode must be justifiable. We first tested

$$
d_{0}=g
$$

for the two processes: one is the evolution of a single source point and the other is that of a single void. For the former, which simulates the probability distribution of a single Brownian particle, or equivalently the fate of an assembly of many Brownian particles, acting as a source of mass transport, the following initial condition has been chosen: $X_{1000}^{0}=10000$ and $X_{i}^{0}=0(i \neq 1000)$ with $g=$ $d_{0}=0.2, N_{x}=2000$, and $N_{t}=10000$. Since $X_{i}^{k}$ 's take zeroes for alternate $i$ 's, the averages of the neighboring pairs, $\left(X_{i}^{k}+X_{i+1}^{k}\right) / 2$, are plotted after $k$-th iteration as shown in Fig. 3(a). Row (I) is the result with sink. When $k$ takes $0,2500,5000,7500$, and 10000 , the initial peak height decreases from 3000 to $81,71,0$, and 0 . The last two zeroes may suggest that the electrode acts as an absorbing wall,

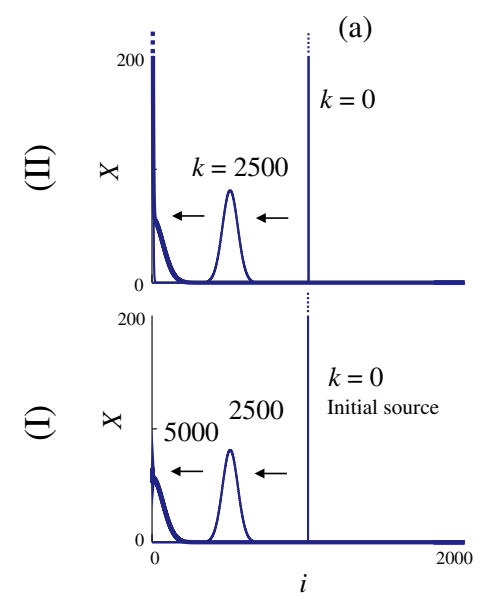

(b)

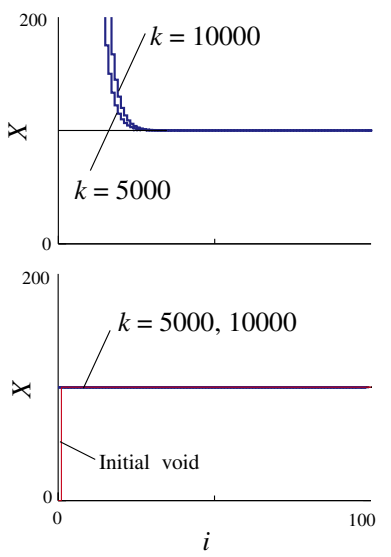

Figure 3. (Color online) Convective diffusion for $g=0.2$. (a) Single point-source. (b) Single void. (I) With sink. (II) Without sink.

but it should be understood that the Brownian particles are carried away with the flow toward the sink. Row (II) is the result without sink, $d_{0}=0$, and $g$ is still kept at 0.2. The peak height changes from 3000 to $81,1395,2620$, and 2620 . The behavior should be understood as suggesting that the Brownian particles accumulate on the electrode.

For the latter case of a void on the surface, the following initial condition has been set up: $X_{0}^{0}=0$ and $X_{i}^{0}=100(i>0)$ with $g=0.2, N_{x}=100$, and $N_{t}=10000$. Since the void is a region in which no particles exist, mass transport should occur toward the surface. The results for the case with the sink, $d_{0}=g=0.2$, are shown in Row (I) of Fig. 3(b) after iterations of $k=0,5000$, and 10000. The distribution eventually becomes flat, meaning that the initial void has disappeared completely. If the sink is detached from the cell at $i=0$, the particles accumulate steadily on the electrode, as shown in Row (II) of Fig. 3(b).

Thus, the test has shown for $g=0.2$ that the same $g$ should be chosen for both of $d_{0}$ and the transport parameters for all $i$ 's. It has been also confirmed that the same applies to other values for $g \leq 1$, and therefore, the importance of the sink has been demonstrated unequivocally.

In order to establish the algorithm, let us pose another question as to whether the previous parameters, $p_{0}=q_{0}=1 / 2$, are really inappropriate for the cell contacting the electrode. Thus, numerical calculations have been carried out for a void on the surface with $g=0.1,0.2,0.5$, and 0.8 for $i \geq 1$, whereas the sink parameter follows the relation $d_{0}=g$. The results are shown in Fig. 4, where the extra accumulation occurs for all the values of $g$, unlike Fig. 3(b)(I). Thus, we can say that $p_{0}$ and $q_{0}$ should also obey the formulas, Eqs. (18) and (19).

\section{Results and Discussion}

\subsection{Results of electrochemical calculations}

Let us simulate the chronoamperometry based on the model proposed in the preceding section. It is assumed that the model developed for the scalar $X$ can be equally applied to the vector $\boldsymbol{P}$. Calculations have been made for the electrode potential $E=-700$ $\mathrm{mV}$, i.e., $E=E_{\mathrm{eq}}-200 \mathrm{mV}$, at which only reduction reaction should occur. In other words, as soon as the potential is applied, Ox begins to decrease near the electrode whereas Rd produced there does not participate in the backward reaction. The current is determined from imbalance between the electron transfer and the mass transport.

For the transition probabilities, the reaction was assumed to proceed symmetrically, i.e., $K_{\mathrm{RO}}=1$, as mentioned above. The 


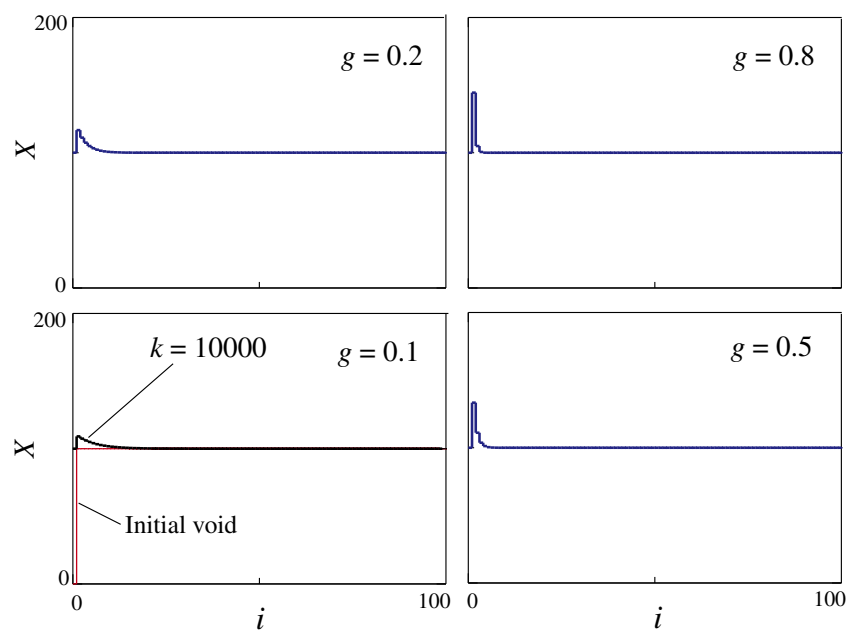

Figure 4. (Color online) Calculations of $X$ similar to Fig. 3(b)(I) with $k=10000$ but $p_{0}=q_{0}=1 / 2$.

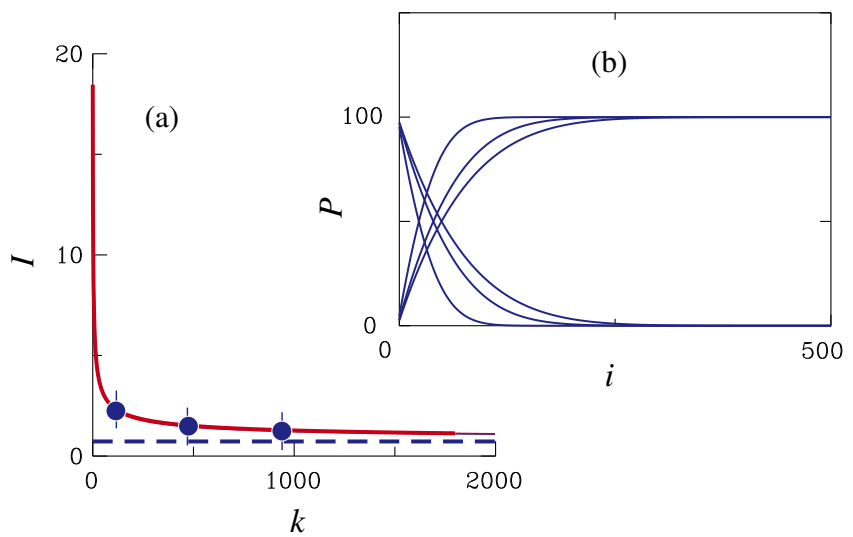

Figure 5. (Color online) Effects of uniform flow on the electrochemical process for $g=0.005$. (a) Current vs. time. Broken line: $I_{\mathrm{S}}$. (b) Concentration vs. distance at the points marked in (a).

enhancement factor $v$ was usually set to unity unless otherwise mentioned. $N_{\text {step }}$ was kept at 16 , that is, a data point was retrieved after 16 iterations, while the number of time steps was $N_{t}=2000$. The space size was set to $N_{x}=2000$, which was so large that the concentration distribution in this region and beyond was hardly affected by mass transport. The initial condition for the concentration was

$$
\boldsymbol{P}_{i}^{0}=\left(\begin{array}{c}
P^{0} \\
0
\end{array}\right), \quad(i=0,1,2, \ldots)
$$

where $P^{0}$ is the initial concentration of $\mathrm{Ox}$, and is set to 100 throughout this paper.

Figure 5 shows the result for $d_{0}=g=0.005$ and $v=1$, where Fig. 5(a) is the plots of the current against time, and the circles indicate the time at which the concentration profile is examined, as in Fig. 5(b). The current was still decreasing in the end of calculation at $k=2000$, and the following model function was employed to get the real value of the stationary-state current.

$$
I^{k}=\frac{1}{\sqrt{a+b k}}+I_{\mathrm{S}}
$$

In Fig. 5, the fitted curve and original one are practically indistinguishable.

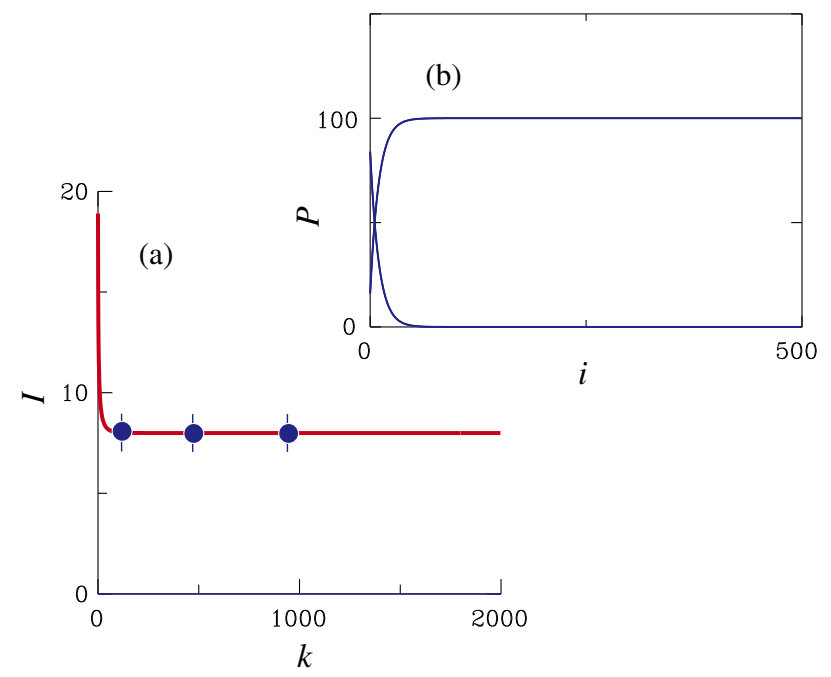

Figure 6. (Color online) Same as Fig. 5 but $g=0.05$.

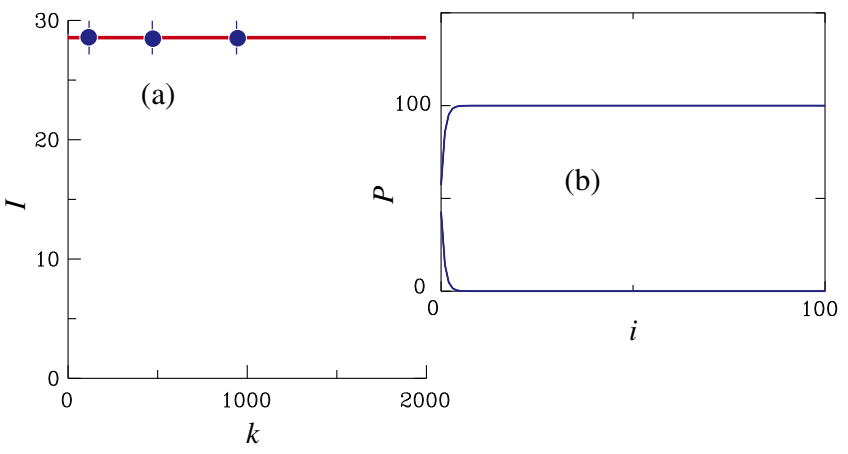

Figure 7. (Color online) Same as Fig. 5 but $g=0.5$.

The results for $g=0.05$ and 0.5 are shown in Figs. 6 and 7, respectively, with $v$ set to unity. Since the currents readily take the stationary values, those at the end point $k=2000$ have been chosen as $I_{\mathrm{S}}$. As $g$ increases, $I$ increases more rapidly near the origin and reaches quickly to the stationary value, as evidenced by the decreasing number of the concentration profiles that can be distinguished. Note that the space range is reduced to one hundred for $g=0.5$.

The whole results are summarized in Fig. 8, where the stationarystate currents are plotted against $g$. The results for $v=0.1$ are also shown. The curves pass through the origin, since $g=0$ gives rise to the Cottrel current, which decays as $k^{-1 / 2}$. The figure shows that the increase in flow velocity is more effective for faster electron-transfer rates; the stationary-state current levels off at relatively slow velocity, if the rate is small.

Since $I_{\mathrm{S}}$ originates from two causes, electron transfer and mass transport, let us plot the reciprocals. The results are shown in Fig. 9, and the data points are aligned along lines if those with small $g$ values are ignored. Regression analysis yields the intercepts 0.0250 for $v=1$ and 0.205 for $v=0.1$. Their reciprocals, 40.0 and 4.88 , are just the limiting currents in Fig. 8, and the ratio of these values is less than that of $v$ 's. This difference will be discussed with reference to Fig. 10 below, where a ten-fold increase in $v$ leads to a less increase in $I_{\mathrm{S}}$. The analysis here may be understood as a 1D-ECA version of the Koutecký-Levici plot, ${ }^{11}$ in which the intercept is the reciprocal of the so-called kinetic current. The present $g$ parameter may be compared to the square root of the rate of revolution, $\sqrt{\omega}$.

The effect of the enhancement factor $v$ is shown in more detail in Fig. 10, where $v$ ranges between 0.01 and 1. For small values of $g$, 


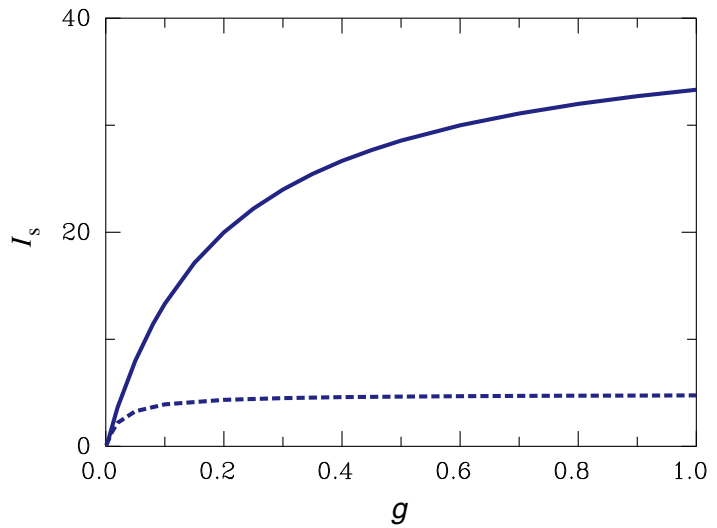

Figure 8. (Color online) Stationary current plotted against asymmetry parameter $g$. Solid line: $v=1$. Broken curve: $v=0.1$.

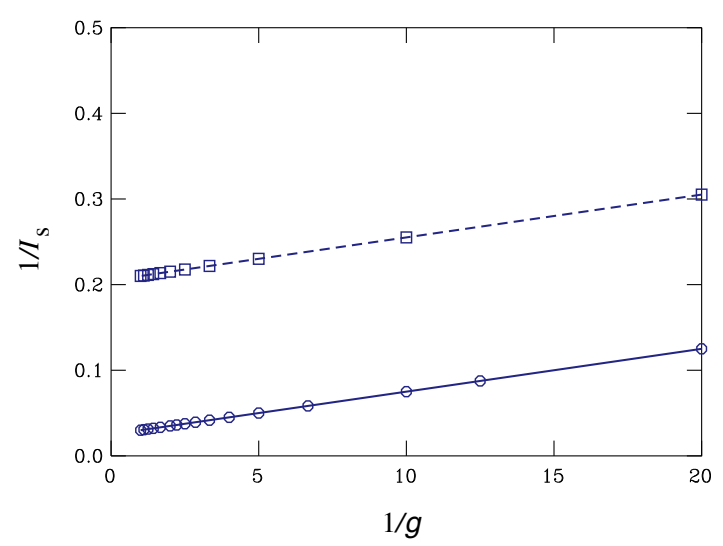

Figure 9. (Color online) Plots of reciprocals for Fig. 8. Solid line: $v=1$. Broken curve: $v=0.1$.

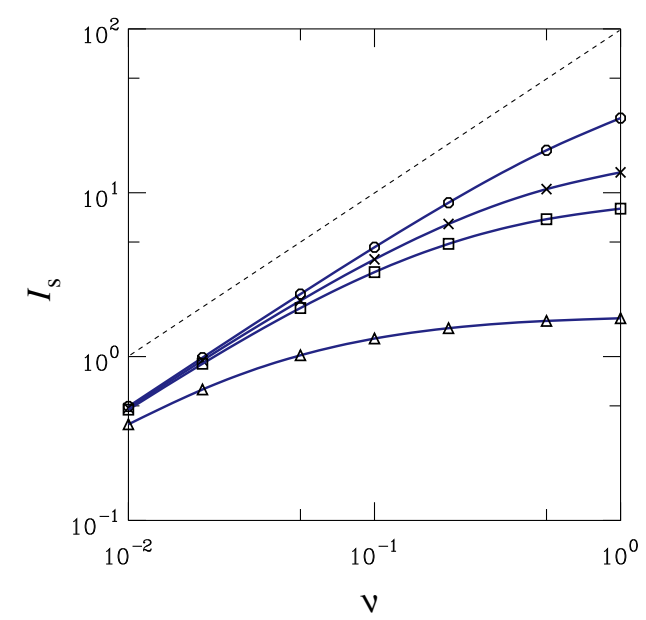

Figure 10. (Color online) Stationary-state current vs. enhancement factor $\nu . \bigcirc: g=0.5 . \times: g=0.1 . \square: g=0.05 . \triangle: g=0.01$. Dotted line: slope of unity.

$I_{\mathrm{S}}$ slowly increases with $v$, and as $g$ increases, the graph of $I_{\mathrm{S}}$ tends to a line with a slope of unity. Note that $I_{\mathrm{S}}$ vanishes for $g=0$.

\subsection{Discussion}

The present model is characterized by a sink for mass transport and asymmetric propagation probabilities, thus mimicking an axial flow toward the electrode. As a matter of fact, this type of flow may be difficult to realize in the real electrochemistry; a typical experiment that utilizes an axial flow is to use RDE, in which the velocity profile varies with both of the distance from the electrode and the angular velocity of revolution, $\omega$. It must be worthwhile to see how the real flow is simplified in the present 1D-ECA (see also Fig. 2). For this purpose, let us review briefly the velocity profile of RDE. ${ }^{11}$ The flow velocity $V_{x}$ along the axis is a quadratic function of the distance from the electrode surface $x$,

$$
V_{x} \propto \omega^{3 / 2} \tilde{v}^{-1 / 2} x^{2} \quad(x \rightarrow 0)
$$

where $\tilde{v}$ is the kinematic viscosity. The quadratic form disappears near the layer boundary, $\delta_{B}$, where

$$
\delta_{B} \propto \omega^{-1 / 2} \tilde{v}^{1 / 2} .
$$

Beyond this distance the velocity tends to a constant value given by

$$
V_{x} \propto \omega^{1 / 2} \tilde{v}^{1 / 2}
$$

The radial velocity $V_{r}$ is a linear function of $x$ in the neighborhood of the surface,

$$
V_{r} \propto \omega^{3 / 2} \tilde{v}^{-1 / 2} r x \quad(x \rightarrow 0)
$$

and tends to vanish in the bulk region. Here $r$ is the distance from the axis. Thus, the layer boundary is regarded as existing in the cell at $i=0$. In the present one-dimensional model the axial velocity is considered as constant, but no particular attention is paid to the streamlines near the electrode surface. Instead, the effect of the bent streamlines on the concentration profile is dealt with by the sink, which is a hypothetical entity.

In spite of the simplicity, however, the present model reveals an essential feature of the flow, since finite stationary-state current is observed and since plots of the reciprocals are meaningful. It is hoped that the model may serve as a prototype of a kind of electrochemistry wherein transport phenomena play a significant role.

As for the finite stationary-state current, it originates not only from flow, but also from stationary distributions of Ox without flow. For example, supplying $\mathrm{H}_{2}$ and $\mathrm{O}_{2}$ to the fuel cell may be regarded as such. If the diffusion length is made equal to $N_{x}$, the initial and boundary conditions, respectively, can be expressed as follows:

$$
\begin{array}{ll}
P_{i}^{0}(\mathrm{Ox})=P^{0} & \left(0 \leq i \leq N_{x} ; k=0\right) \\
P_{i}^{k}(\mathrm{Ox})=P^{0} & \left(i \geq N_{x} ; 0 \leq k \leq N_{t}\right)
\end{array}
$$

In the limit of $k \rightarrow \infty$ the concentration profile becomes linear. For finite values of $k$ the flux should be inversely proportional to the diffusion length and, if electron transfer is fast enough, so is the stationary-state current. The results of numerical calculation are shown in Fig. 11, where $N_{x}$ is varied between 200 and 1000. The behavior for $v=1$ yields a linear relation between $I_{\mathrm{S}}$ and $N_{x}^{-1}$. For the electrode with $v=0.01$, the current is smaller and a linear relation does not hold any more.

From a view point of the evaluation of catalytic activity, Fig. 11 is not of much use, since an increase in activity $v$ by two orders of magnitude gives rise at most to a two-fold increase. Thus, the stationary-state current is not necessarily a good measure of catalytic activity. The difficulty can be overcome using the procedure that can characterize active area of catalyst, ${ }^{12}$ for which RDE is often employed.

\section{Conclusion}

A one-dimensional uniform flow has been successfully implemented in 1D-ECA by way of a sink and asymmetric propagation probabilities, both of which are characterized by probability parameters related to the flow velocity. A uniform flow as well as a finite gradient of diffusing reactants leads to finite stationary-state currents. 


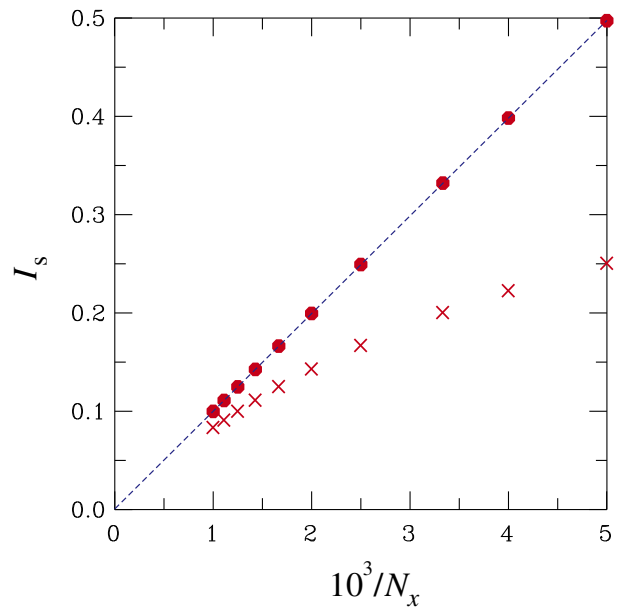

Figure 11. (Color online) Stationary current plotted against $10^{3} /$ (diffusion length). $I_{\mathrm{S}}$ for $v=1 . \times: I_{\mathrm{S}}$ for $v=0.01$. Broken line: fitted to data points with slope $=0.09938$, intercept $=0.00063$.

\section{Acknowledgments}

The author is grateful to Dr. G. Samjeske of the Innovation Research Center for Fuel Cells, the University of ElectroCommunications, for helpful discussion.

\section{References}

1. See, for example, C. A. Angelucci, M. D’Villa Silva, and F. C. Nart, Electrochim. Acta, 52, 7293 (2007).

2. A. J. Bard and L. R. Faulkner, Electrochemical Methods. Fundamentals and Applications, 2nd ed., John Wiley and Sons, New York, NY, p. 163 (2001).

3. S. Hayashi, Electrochemistry, 81, 16 (2013).

4. S. Hayashi, Electrochemistry, 81, 269 (2013); [correction] 81, 572 (2013).

5. S. Hayashi, Electrochemistry, 81, 688 (2013).

6. S. Hayashi, Electrochemistry, 81, 961 (2013).

7. S. Hayashi, Engineer no Tame no Denki-Kagaku (Electrochemistry for Engineers), Coronasha, Tokyo, p. 146 (2012). [in Japanese]

8. M. Kac, Selected Papers on Noise and Stochastic Processes (Ed. N. Wax), Dover, New York, NY, p. 295 (1954); M. Kac, Am. Math. Monthly, 54, 369 (1946)

9. A. J. Bard and L. R. Faulkner, Electrochemical Methods. Fundamentals and Applications, 2nd ed., p. 785 (2001).

10. T. Oosawa, K. Kanou, and S. Kuwabata, Basic Electrochemistry, Kagaku-Dojin, p. 126127 (2000). [in Japanese]

11. A. J. Bard and L. R. Faulkner, Electrochemical Methods. Fundamentals and Applications, 2nd ed., p. 335 (2001).

12. See, for example, I. Takahashi and S. S. Kocha, J. Power Sources, 195, 6312 (2010). 